\title{
Physician and Staff Acceptance of Care Managers in Primary Care Offices
}

\author{
Jean M. Malouin, MD, MPH, Rebecca A. Malouin, PhD, MPH, MS, \\ Issidoros Sarinopoulos, PhD, Marie Beisel, MSN, RN, CPHQ, \\ Diane Bechel-Marriot, DrPH, Amanda First, MHSA, Ginger M. Gamble, MPH, \\ and Clare Tanner, $P h D$
}

Introduction: Embedded care managers are increasingly implemented as part of the care team within primary care practices, yet previous studies have indicated variability in acceptance by physicians and staff. This study assesses the acceptability of care managers among staff and physicians within the Michigan Primary Care Transformation (MiPCT) demonstration.

Methods: Care manager acceptance was measured using a web-based survey distributed to practices participating in the MiPCT demonstration. Results: Both physicians and staff reported high levels of care manager acceptance. Longer length of care manager employment at the practice, higher care manager FTE dedicated to care management, and care manager employed by practice were all significantly associated with care manager acceptance.

Discussion: The MiPCT demonstration found high care manager acceptance across all care team members. The high level of acceptance may be due to the structures and processes developed by MiPCT to support implementation of care managers and the length of the intervention period.

Conclusion: The MiPCT demonstration confirms that following three years of implementation, embedded care managers are acceptable to both physicians and staff within primary care practices. Importantly, embeddedness, or the amount of time care managers are located within practices, is associated with increased acceptance. (J Am Board Fam Med 2017;30:140-149.)

Keywords: Michigan, Patient Care Management, Patient Care Team, Primary Health Care, Surveys and Questionnaires

Care management is increasingly incorporated within new team-based models of primary care. Care management is defined as "the application of systems, science, incentives and information to improve medical practice and assist consumers and their support systems to become engaged in a collaborative process designed to manage medical, social and mental health conditions more effec-

This article was externally peer reviewed.

Submitted 1 August 2016; revised 17 November 2016; accepted 22 November 2016.

From the Department of Family Medicine, University of Michigan, Ann Arbor (JMM); the Department of Family and Community Medicine, Michigan State University, East Lansing (RAM); the Center for Data Management and Translational Research, Michigan Public Health Institute, Okemos (IS, GMG, CT); the Michigan Care Management Resource Center, University of Michigan, Ann Arbor (MB); and MiPCT, University of Michigan, Ann Arbor (DB-M, AF). tively." 1 The specialized role of a care manager within the primary care setting, referred to as an embedded care manager if located within the practice, is often performed by nurses, social workers, nurse practitioners, or physician assistants. Care managers use registries to identify high-risk patients for proactive management, provide evidence-based care using shared decision-making, use collaborative practice models, provide patient

Funding: The MiPCT evaluation is supported by administrative funds provided by the participating payers, including the Centers for Medicare and Medicaid Services (Medicare), the Michigan Department of Community Health (Medicaid), Blue Cross Blue Shield of Michigan, Blue Care Network, and Priority Health.

Conflict of interest: none declared.

Corresponding author: Jean M. Malouin, MD, MPH, Department of Family Medicine, University of Michigan, 1150 West Medical Center Dr, M7300 Medical Science I, Ann Arbor, MI 48109-5625 (E-mail: jskratek@med.umich.edu). 
self-management support, and assist with care coordination and transitions between care settings. ${ }^{2,3}$

Several small studies have found that care management within primary care practices increases engagement and improves outcomes for patients with diabetes and other chronic conditions. ${ }^{4-6} \mathrm{Em}$ bedded care managers are increasingly used in new models of primary care, as evidenced in several national and regional patient-centered medical home and primary care payment demonstrations. ${ }^{7,8}$ For example, an early study of the Centers for Medicare and Medicaid Services Comprehensive Primary Care Initiative found that $24 \%$ of the practices reported having an embedded care manager on staff. Within the practices recognized as patientcentered medical homes, $>40 \%$ of the practices reported having an embedded care manager on staff. $^{7}$

While recognizing the benefits of embedded care management, the process of introducing a new role within a primary care setting involves substantial planning and design, including attention to professional development, role definition, and the care team. ${ }^{9,10}$ In short, care management must be operationalized in a manner embraced by physicians and other care team members. Smaller studies of care manager implementation have found that clinical staff within primary care practices support having onsite or embedded care managers, as opposed to case management by off-site health plan or disease management companies, as the most effective form of patient engagement in disease management. ${ }^{11,12}$ However, studies of the early implementation of embedded care managers have identified physician and staff concerns that the care managers interfere with their work. ${ }^{13}$ Others have found that while many physicians and staff members appreciate the relationship between the care manager and patients, some felt threatened by a perceived weakening of their own relationship with patients. ${ }^{8}$ Many of these studies followed physicians and staff for only a short period of time and included only a small number of practices within the sample. ${ }^{8,11-13}$ The objectives of the current study were twofold: (1) to assess the acceptability of care managers among staff and physicians within primary care practices across a large, state-wide demonstration program, and (2) to evaluate whether the amount of time a care manager spends physically located within a practice (level of care manager embedment) is predictive of staff and physician acceptability after adjusting for a variety of survey respondent and practice characteristics.

\section{Methods}

\section{Setting: The Michigan Primary Care Transformation Demonstration}

The Michigan Primary Care Transformation (MiPCT) Demonstration, a Centers for Medicaid and Medicare Multi-Payer Advanced Primary Care Practice demonstration, offers an opportunity to study the largest implementation of embedded care managers in patient-centered medical homes in the United States. The MiPCT demonstration includes 347 practices and $>1500$ physicians, enabling a representative evaluation of physician and staff experience with embedded care managers. The MiPCT demonstration uses standardized training models, documentation tools, and a hierarchy of trainers, including master trainers and clinical leads. Practices are paid $\$ 3.00$ per member per month for Medicaid patients, $\$ 4.50$ per member per month for Medicare beneficiaries (because of the higher prevalence of disease burden and comorbidities among the elderly population), and fee-for-service encounter-based codes for commercial patients. MiPCT hired and trained $>600$ care managers, the majority of whom are licensed registered nurses. Of the $>400$ current care managers (200 were lost to turnover), 92\% are embedded within primary care practices, meaning that they are physically based in those practices for the majority of their time as care managers. Other care managers are employed by a provider organization or health system and shared across multiple practices. The demonstration requires 2 care managers for every 5000 MiPCT patients within a provider organization, and each care manager is expected to have an average caseload of 125 to 150 patients.

To build relationships between care managers, physicians, and staff within participating practices, the MiPCT demonstration leadership used a multipronged approach to change culture. The demonstration required practices to provide workspace and resources for care managers, ensure adequate staffing sufficiency for the care manager, and engage in team-based activities (eg, team huddles, medical home meetings), as outlined in an annual practice participation agreement. Furthermore, the demonstration leadership sponsored town hall din- 
ners where $>50$ physician and care manager dyads were presented stories of peer team-based care success, shared best practices, and discussed ways to overcome workflow challenges in implementing embedded care managers. In addition, the demonstration sponsored practice learning collaboratives with 47 teams and held monthly forum calls open to the entire MiPCT community to discuss questions and generate stakeholder discussion of topics generated by the practice team community. Finally, MiPCT leadership organized annual summits to share demonstration results and honor top-performing practices.

\section{Data Sources}

Physician and Staff Survey

Staff and physician acceptance were measured using a Web-based survey distributed to key practice contacts, with instructions to forward the link to all staff working at MiPCT practices. The survey was administered from August to October 2014, during the third year of the 5-year demonstration. The questions within this study were part of a larger survey of physicians, other providers, and practice staff. The specific items related to care managers were developed through a literature review and group discussions with the MiPCT leadership team, and were tested for clarity of wording and appropriate response categories with multiple stakeholder groups and the MiPCT advisory committee.

Provider and staff characteristics, including the number of years worked at the practice and their professional roles, were included in the survey. In addition, because care manager embedment was not the only disruptive change occurring within practices, the survey also included questions about changes in practice leadership, ownership, and use of electronic health records within the past year.

\section{Project Administrative Data}

Most care manager embedment measures originate from practice-specific reports, which are required as part of MiPCT program monitoring. Each quarter, care manager implementation information by practice is reported through an online portal; the information include the number of care managers, dates of hire, care manager employer, total care manager percentage full-time equivalent (FTE) allocation, and primary location of care manager (practice or a central location). To match the time frame of the provider and staff survey, this article reports care manager documentation from the third quarter of 2014. The number of care managers, average care manager longevity, and care manager employer variables are derived directly from this report. The average embedded care manager FTE variable is derived by dividing the total embedded care manager FTE allocation by the total number of care managers in each practice. As such, this variable is a measure of the amount of time care managers spend embedded within the practice. Because we use the average rather than the total care manager FTE, this measure is independent of the total number of care managers within a practice, a number that is dependent on patient volume and practice size. Finally, the care manager per number of patients variable (a ratio) comes from dividing the quarterly reported care manager FTE by the number of attributed patients in each practice as reported by participating payers.

Additional practice covariates were derived from provider files maintained for project administration. The provider files link each participating practice to provider names and other identifiers. The practice size variable is calculated as the number of providers associated with a practice. Patient data are obtained monthly from participating payers and maintained within the MiPCT administrative database. Pediatric practices are defined as those in which $80 \%$ of patients are aged $<18$ years.

\section{Data Linkages}

MiPCT administration assigns identification numbers to each participating practice. Providers are identified by their national provider identifier and are associated in the administrative database with both patients and practices. For this study, variables constructed from MiPCT administrative data were aggregated to the practice level and merged into the survey data files using the practice identification number. Respondents were not identified individually on the survey; however, they were asked to select their provider organization and practice from a drop-down list.

\section{Care Manager Measures and Analyses}

We provide summary statistics on the level of care manager acceptance as expressed by staff and physician measures. Four questions about the acceptability of care management were asked of both physicians and staff members. An additional 3 ques- 
tions were asked of physicians, for a total of 7 . All items were scored on a 5 -point scale. Having hypothesized single dimensions for the staff and physician acceptance measures, the construction of the physician and staff composite scales was assessed using a single-factor confirmatory factor analysis. The use of factor analysis for this survey was supported by the Barlett test of sphericity and KaiserMeyer-Olkin index measures of sampling adequacy. Single items were then averaged together to create composite measures of staff and physician acceptance.

Separate analyses were subsequently conducted to predict physician and staff acceptance based on the level and nature of care management embedment, as well as respondent demographic and practice characteristics. The demographic characteristics of age and sex were included as predictors in the multivariate model for both staff and physician analyses. Additional variables were determined based on bivariate analyses, which tested the association between each variable and care manager acceptability. The decision to include a variable from the multivariate model was based on the 2 -sided $P$ value of a general linear model test. Variables that failed to meet criteria included a categorical variable of practice ownership and binary variables indicating potential disruption, such as practice leadership change, high provider turnover, and high staff turnover.

The resulting list of predictors from the survey respondent, practice, and care manager embedment categories were included in each analysis; the only exception was the inclusion of professional role as a predictor for staff, but not physician, acceptance analysis. Predictors were submitted to a mixed-model analysis, with practice as a random factor, using the mixed-effects model procedure with post hoc comparisons in SPSS software. The major capabilities of this procedure include handling correlated data and unequal variances, as well as complex situations in which data units are nested, as in our case, where several survey respondents could be reporting from the same practice.

\section{Results}

\section{Respondent and Practice Demographics}

A total of 320 physicians and 1137 other staff members (including care managers) responded to the survey. At that time, 1586 physicians and approxi- mately 4949 nonphysician staff members were engaged with MiPCT. These data represent 986 respondents from 237 practices $(68 \%$ of 347 MiPCT practices), including 36 of the $45 \mathrm{MiPCT}$ pediatric practices. After excluding care managers and incomplete or invalid surveys, the final sample consisted of 284 physicians and 699 staff responses, resulting in an estimated response rate of $20 \%$ for physicians and $23 \%$ for staff members. To explore potential nonresponse bias, we compared practice and model implementation characteristics among practices that were included in the final sample and those that were not. Results showed that there were no significant differences between responding and nonresponding practices $(P>.09)$.

The professional roles of the respondents varied and were divided fairly equally into thirds among physicians, clinical staff, and administrative staff. Of note, $17.6 \%$ were medical assistance and $18.7 \%$ were other administrative professionals. The majority of respondents worked in practices with 1 or 2 embedded care managers; $46 \%$ of respondents came from practices in which the care managers have served for $\geq 2.5$ years, on average. As noted previously, care manager FTE was evenly distributed within the practices represented in this survey. Only $20 \%$ of respondents worked in practices in which care managers were directly employed by the practice. Survey respondent, practice, and model implementation characteristics can be viewed in Table 1.

\section{Staff and Physician Acceptance}

It was first determined that the survey data were appropriate for analysis using factor analysis. The significance of the Bartlett test was $<.05$, and the Kaiser-Meyer-Olkin index was higher than .7, thus meeting the criteria of appropriateness to apply factor analysis. Using principal component factor analysis, the resulting factor loading matrix was varimax rotated. The criterion to decide on the number of factors was an eigenvalue $>1$, which was fulfilled by a single factor for each acceptance measure. The single factor explained $81.5 \%$ and $70.1 \%$ of total variance for the staff and physician measures, respectively. The reliability for both measures is excellent (Cronbach $\alpha=0.92$ for both).

Analysis of survey responses revealed a high level of acceptance for both staff and physician measures, with composite average scores of $4.0 \pm 0.79$ and $4.2 \pm 0.73$, respectively. Among respondents, 
Table 1. Practice, Model Implementation, and Respondent Characteristics of Physicians and Practice Staff Responding to a Survey Conducted During the Third Year of the 5-Year Demonstration

\begin{tabular}{|c|c|c|c|c|}
\hline & \multicolumn{2}{|c|}{ Staff $(\mathrm{n}=699)$} & \multicolumn{2}{|c|}{ Physicians $(\mathrm{n}=284)$} \\
\hline & No. & $\%$ & No. & $\%$ \\
\hline \multicolumn{5}{|l|}{ Respondent characteristics } \\
\hline \multicolumn{5}{|l|}{ Age (years) } \\
\hline $18-29$ & 113 & 16.2 & 6 & 2.1 \\
\hline $30-39$ & 168 & 24.0 & 51 & 18.0 \\
\hline $40-49$ & 156 & 22.3 & 84 & 29.6 \\
\hline $50-59$ & 180 & 25.8 & 88 & 31.0 \\
\hline$\geq 60$ & 78 & 11.2 & 55 & 19.4 \\
\hline Female sex & 669 & 95.7 & 145 & 51.1 \\
\hline Professional role & & & \multicolumn{2}{|c|}{ NA } \\
\hline Nurse practitioner/physical assistant & 49 & 7.0 & & \\
\hline Other nurse & 100 & 14.3 & & \\
\hline Medical assistant & 174 & 24.9 & & \\
\hline Practice manager & 136 & 19.5 & & \\
\hline Other clinical & 46 & 6.6 & & \\
\hline Other administrative & 194 & 27.8 & & \\
\hline Job longevity ( $\geq 7$ years)* & 317 & 45.4 & 187 & 65.8 \\
\hline \multicolumn{5}{|l|}{ Practice characteristics } \\
\hline Pediatric practice & 96 & 13.7 & 34 & 12.0 \\
\hline \multicolumn{5}{|l|}{ Practice size (no. of providers) } \\
\hline $1-2$ & 161 & 23.0 & 39 & 13.7 \\
\hline $3-4$ & 170 & 24.3 & 63 & 22.2 \\
\hline $5-7$ & 196 & 28.0 & 71 & 25.0 \\
\hline $8-11$ & 104 & 14.9 & 51 & 18.0 \\
\hline 12 or more & 68 & 9.7 & 60 & 21.1 \\
\hline EHR implementation change & 135 & 19.3 & 116 & 40.8 \\
\hline \multicolumn{5}{|l|}{ Model implementation characteristics } \\
\hline Average CM job longevity (2.5 years or more)* & 315 & 45.1 & 135 & 47.5 \\
\hline \multicolumn{5}{|l|}{ Average embedded CM FTE } \\
\hline$<25$ & 104 & 14.9 & 32 & 11.3 \\
\hline $25-49$ & 134 & 19.2 & 60 & 21.1 \\
\hline $50-74$ & 160 & 22.9 & 54 & 19.0 \\
\hline $75-99$ & 125 & 17.9 & 59 & 20.8 \\
\hline 100 & 173 & 24.7 & 78 & 27.5 \\
\hline \multicolumn{5}{|l|}{ Embedded CMs (n) } \\
\hline 0 & 27 & 3.9 & 12 & 4.2 \\
\hline 1 & 318 & 45.5 & 126 & 44.4 \\
\hline 2 & 194 & 27.8 & 86 & 30.3 \\
\hline 3 or 4 & 108 & 15.5 & 37 & 13.0 \\
\hline$\geq 5$ & 52 & 7.4 & 23 & 8.1 \\
\hline $\mathrm{CM}(\mathrm{s})$ employer is the practice & 161 & 23.0 & 37 & 13.0 \\
\hline Meeting or exceeding patients'/CM standards & 375 & 53.6 & 172 & 60.6 \\
\hline
\end{tabular}

*Distributed approximately normally and treated as a continuous variable in the analysis.

CM, care manager; EHR, electronic health record; FTE, full-time equivalent; NA, not applicable.

$70.4 \%$ of nurses and $68.9 \%$ of physicians rated the acceptability an average of 4 or higher on a 5-point acceptance scale. Individual item results for each measure are presented in Table 2 . The effect of the care manager-to-number of patients ratio was tested using both the actual number and a categorical form of the variable (with thresholds at 1200, 2400, 3600, and 4800 patients per care 
Table 2. Item-Level and Composite Measures of Model Acceptance by Staff and Physicians in MiPCT Practices

\begin{tabular}{|c|c|c|c|c|}
\hline & \multicolumn{2}{|c|}{ Staff $(n=699)$} & \multicolumn{2}{|c|}{ Physicians $(\mathrm{n}=284)$} \\
\hline & Mean & $\mathrm{SD}$ & Mean & $\mathrm{SD}$ \\
\hline \multicolumn{5}{|l|}{ Single item* } \\
\hline The $\mathrm{CM}$ is an important member of our team. & 4.07 & 0.88 & 4.13 & 0.98 \\
\hline The CM improved our ability to meet patient needs. & 4.08 & 0.87 & 4.14 & 0.95 \\
\hline There is good CM-staff communication. & 3.88 & 0.93 & 4.04 & 0.97 \\
\hline MiPCT has been a positive experience. & 3.97 & 0.85 & 4.05 & 0.89 \\
\hline I have complete trust in CMs. & & & 4.47 & 0.76 \\
\hline I refer eligible patients to CMs. & & & 4.08 & 0.93 \\
\hline I would like care management to continue. & & & 4.51 & 0.77 \\
\hline \multicolumn{5}{|l|}{ Composite } \\
\hline CM acceptance & 4.00 & 0.80 & 4.20 & 0.73 \\
\hline
\end{tabular}

*All items were scored on a scale of 1 to 5 .

CM, care manager; MiPCT, Michigan Primary Care Transformation; SD, standard deviation.

manager). For the analysis of staff acceptability, results showed that neither the actual number nor the categorical version were significant predictors $(P>.36$ for both). For the analysis of physician acceptability, the actual number variable approached significance $(P=.065)$, although the categorical variable was not significant $(P>.30)$.

Analyses to determine the association between survey respondent, practice, and model implementation characteristics and acceptance resulted in largely consistent results for staff and physician acceptance measures. Mixed-model analysis results presented in Table 3 indicate that average embedded care manager FTE $(P=.002)$ and average care manager job longevity $(P=.028)$ were significant predictors of greater staff acceptance. However, a single variable from the practice characteristics category, pediatric practice $(P=.002)$, was associated with lower staff acceptance. From the survey respondent characteristics category, respondents' professional role $(P<.001)$ was also a significant predictor of staff acceptance. Post hoc comparisons (data not shown) revealed that this effect was primarily driven by lower acceptance among medical assistants and respondents in the "other administrative role" category.

Consistent with the staff acceptance analysis, average embedded care manager FTE $(P<.001)$ and care manager job longevity $(P=.018)$ were associated with greater physician acceptance. In addition, a third predictor from the model implementation category, the practice as the care manager's employer $(P=.013)$, was also associated with greater physician acceptance. Clustering at the practice level contributed significantly in both staff $(P<.001)$ and physician $(P=.029)$ mixed-model analyses, indicating significant concordance across respondents within the same practice.

\section{Care Management Embedment and Acceptance}

Intensity of contact with a care manager within a practice was found to be associated with greater acceptability by physicians and staff. Specifically, care manager job longevity and average embedded care manager FTE correlated positively with both staff and physician acceptance. Results indicate that staff and provider acceptance increased with higher average care manager FTE embedment in a roughly linear fashion (Figure 1). Interestingly, the practice as a care manager's employer was highly correlated with physician acceptance but not staff acceptance. The number of embedded care managers was not significant to either staff or physician acceptance.

\section{Discussion}

The MiPCT demonstration confirms that after 3 years of an intervention, embedded care managers are acceptable to both physicians and staff within primary care practices, regardless of practice size or specialty. Both physicians and practice staff indicated that the embedded care manager is an important member of the team and improved the team's ability to meet patient needs.

Unlike previous studies that found varying acceptance of embedded care managers by physicians and staff, ${ }^{8,13}$ this study found very high acceptance 
Table 3. Results of Mixed Model Analysis with Care Manager Acceptance as the Dependent Variable and Respondent Characteristics, Practice Characteristics, and Model Implementation as Fixed-Effect Independent Variables

\begin{tabular}{|c|c|c|c|c|}
\hline \multirow[b]{2}{*}{ Predictors } & \multicolumn{2}{|c|}{ Staff Acceptance } & \multicolumn{2}{|c|}{ Physician Acceptance } \\
\hline & $\beta$ & $P$ Value & $\beta$ & $P$ Value \\
\hline \multicolumn{5}{|l|}{ Respondent characteristics } \\
\hline \multicolumn{5}{|l|}{ Age, years (reference: $50-59$ ) } \\
\hline $18-29$ & 0.23 & .013 & 0.29 & .376 \\
\hline $30-39$ & 0.07 & .365 & 0.28 & .055 \\
\hline $40-49$ & 0.09 & .259 & 0.24 & .037 \\
\hline$\geq 60$ & 0.02 & .809 & 0.03 & .798 \\
\hline \multicolumn{5}{|l|}{ Sex (reference: female) } \\
\hline Men & -0.12 & .402 & 0.03 & .695 \\
\hline Professional role (reference: other administrative) & & & \multicolumn{2}{|c|}{ Excluded/NA } \\
\hline Nurse practitioner/physical assistant & 0.31 & .006 & & \\
\hline Other nurse & 0.20 & .022 & & \\
\hline Medical assistant & -0.09 & .228 & & \\
\hline Practice manager & 0.24 & .035 & & \\
\hline Other clinical & 0.40 & .000 & & \\
\hline Job longevity & 0.01 & .032 & 0.01 & .318 \\
\hline \multicolumn{5}{|l|}{ Practice characteristics } \\
\hline \multicolumn{5}{|l|}{ Pediatric practice (reference: no) } \\
\hline Yes & -0.35 & .002 & 0.07 & .633 \\
\hline \multicolumn{5}{|l|}{ Practice size (reference: $5-7$ ) } \\
\hline $1-2$ & -0.08 & .538 & 0.05 & .792 \\
\hline $3-4$ & -0.07 & .529 & 0.16 & .255 \\
\hline $8-11$ & -0.11 & .390 & -0.07 & .648 \\
\hline$\geq 12$ & -0.11 & .481 & 0.02 & .907 \\
\hline \multicolumn{5}{|l|}{ EHR implementation change (reference: no) } \\
\hline Yes & 0.14 & .156 & -0.08 & .461 \\
\hline \multicolumn{5}{|l|}{ Model implementation characteristics } \\
\hline Average $\mathrm{CM}$ job longevity & 0.13 & .028 & 0.17 & .018 \\
\hline \multicolumn{5}{|l|}{ Average embedded CM FTE (reference: 100) } \\
\hline$<25$ & -0.53 & .000 & -0.59 & .001 \\
\hline $25-49$ & -0.41 & .002 & -0.50 & .001 \\
\hline $50-74$ & -0.29 & .019 & -0.26 & .080 \\
\hline $75-99$ & -0.17 & .159 & -0.09 & .558 \\
\hline \multicolumn{5}{|l|}{ Embedded CMs, n (reference: 1) } \\
\hline 0 & -0.19 & .334 & -0.38 & .120 \\
\hline 2 & 0.12 & .268 & 0.13 & .266 \\
\hline 3 or 4 & 0.26 & .084 & 0.02 & .919 \\
\hline$\geq 5$ & -0.06 & .732 & -0.15 & .463 \\
\hline \multicolumn{5}{|l|}{$\mathrm{CM}(\mathrm{s})$ employer is the practice (reference: no) } \\
\hline Yes & -0.12 & .295 & 0.34 & .013 \\
\hline \multicolumn{5}{|l|}{$\begin{array}{l}\text { Meeting or exceeding patients/CM standard } \\
\text { (reference: no) }\end{array}$} \\
\hline Yes & 0.04 & .667 & -0.13 & .266 \\
\hline
\end{tabular}

Practice was treated as a random effect in the model. Boldface indicates a significant association at $P<.05$.

$\mathrm{CM}$, care manager; EHR, electronic health record; FTE, full-time equivalent; NA, not applicable.

by all staff and physicians across roles and practice types. Because the survey occurred following 3 years of implementation, perhaps time was a factor in the high rates of acceptance, through culture change resulting from communication, defined job roles, and trust. Furthermore, unlike previous stud- 
Figure 1. Adjusted mean acceptance scores by level of CM FTE embedment derived from separate analyses for physicians and staff. Error bars represent standard error of the mean. $C M=$ Care Manager; FTE $=$ Full Time Equivalent.

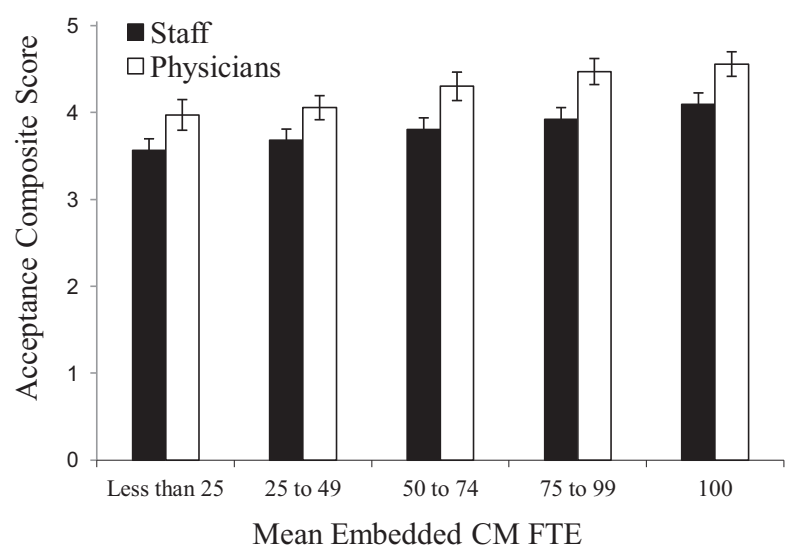

ies, MiPCT leadership implemented infrastructure to support practices and care managers during the implementation process, such as online and Webbased resources and in-person meetings. The supporting infrastructure assisted with sense-making, decision making, planning, and coordinating within the practices, enabling the implementation and ultimate acceptance of embedded care managers.

Factors associated with staff acceptance of embedded care managers include professional role of the respondent, a respondent's longevity within their position, pediatric practice (negative impact), average care manager job longevity, and average embedded care manager FTE. Factors associated with physician acceptance include average care manager job longevity, average embedded care manager FTE, and care manager employment by practice (as opposed to a physician organization, health system, or other entity).

FTE or effort allocation of embedded care managers is fairly equally distributed by quartile among the responding staff and physicians. Interestingly, the average FTE of embedded care manager is highly correlated with care manager acceptance by both staff and physicians, with the higher acceptance associated with higher average FTE. This measure was chosen because it represents the average amount of time a care manager spends located directly within the practice; it is a measure of care manager embedment that is independent of the total number of care managers in the practice and therefore is also not related to practice size.

Staff in pediatric practices were significantly less likely to report acceptability with the care manager role compared with other practices. This may be because pediatric patient needs differ significantly from adult patient needs. Pediatric patients are less likely to suffer from chronic illness than adult patients. ${ }^{14}$ This is consistent with reports from practices that dropped out of the MiPCT demonstration. Pediatric practices were significantly more likely than other practices to drop out of the demonstration, and they reported difficulty in maintaining the 2 care managers per 5000 patient FTE requirement as the top reason for leaving.

\section{Limitations}

One limitation of the study is the low response rates: $20 \%$ for physicians and $23 \%$ for staff members. However, these rates are consistent with other surveys of physicians. ${ }^{15,16}$ The low response rates may have been the result of survey and reporting fatigue, as physicians and staff were asked to respond to many surveys throughout the MiPCT demonstration, both from the MiPCT evaluation and for other administrative and research initiatives in Michigan. ${ }^{17}$ In addition, the MiCPT evaluation team was unable to offer financial incentives. Because the survey was anonymous, it is impossible to fully assess potential differences between respondents and nonrespondents. The analysis of differences in practices represented and not represented in the study indicates no difference in practice characteristics.

In addition, it is not known which of the care managers served as staff members within the practices before the beginning of MiPCT. Some practices hired existing nurses as part- and full-time care managers through MiPCT. Those physicians 
and staff members who knew the care manager before MiPCT may have reported higher acceptability because of familiarity with the individual as opposed to the care manager role.

Finally, although the specific items related to care managers were vetted by multiple stakeholders, the care management scale has yet to be psychometrically validated. Therefore the findings need to be considered with caution.

\section{Conclusions}

Understanding the impact of the average amount of time care managers spend within a practice is believed to be important for a number of reasons. During implementation, practice and provider organization representatives anecdotally reported to MiPCT leadership that when care managers were distributed across multiple practices, or for other reasons were not embedded within practices for significant amounts of time, team communication and referral workflows were difficult to implement. In addition, building trust between the care managers and physicians and practice staff was more difficult. This study provides direct evidence that the average amount of time spent by care managers in a practice (measured by average FTE allocated on location within practices) is indeed related to physician and staff acceptance of this new role. To the extent that physician and staff acceptance of the care manager role is important for achieving better patient outcomes, this study indicates the importance of embedded care management. Future MiPCT studies will test the direct role of embedment in patient outcomes.

MiPCT is the largest assessment of primary care practices regarding their experience with embedded care managers. Most previous studies of care management experience include smaller qualitative studies over a short period of time. This study assesses 3 years of experience with statewide implementation of care managers within 400 primary care practices. The global acceptability of the embedded care manager is likely the result of several core elements of this demonstration. First, the demonstration leadership designed a multipronged approach to support practices and care managers when embedding care managers within the practice, including multiple opportunities to participate in virtual and in-person meetings throughout the demonstration. The transition to team-based care is not "plug and play"; it requires engaged leadership and active facilitation. Second, practices were given funds and required to provide protected time for the care managers. Although some care managers were hired from within the practices, perhaps moving from a traditional nursing role to an embedded care manager role, the work was not in addition to current work, but rather new work with protected time for the expected functions of the care manager. Third, the demonstration provided ongoing training for care managers using a standardized, evidence-based model. The role and day-to-day responsibilities of a care manager are quite different from the traditional role of a practice-based registered nurse or social worker and require specialized training to ensure success. Finally, the demonstration allowed enough time for practice change, both cultural and structural, to enable the implementation of care management and the development of a financial sustainability plan for the embedded care manager. As health systems and practices implement embedded care managers, practice transformation support, financial support for protected care manager time, care manager training, adequate time for the implementation, and a sustainability plan are essential elements for primary care practice acceptance of the embedded care manager role.

To see this article online, please go to: http://jabfm.org/content/ 30/2/140.full.

\section{References}

1. Center for Healthcare Strategies, Inc. Care management definition and framework fact sheet. 2007. Available from: http://www.chcs.org/media/Care_Management_Framework.pdf. Accessed January 22, 2017.

2. MiPCT Demonstration Project. 2011. Available from: http://mipct.org/resources/mipct-general-overview/. Accessed January 22, 2017.

3. Taylor EF, Machta RM, Meyers DS, Genevro J, Peikes DN. Enhancing the primary care team to provide redesigned care: the roles of practice facilitators and care managers. Ann Fam Med 2013;11: 80-3.

4. Hudon C, Chouinard MC, Diadiou F, Lambert M, Bouliane D. Case management in primary care for frequent users of health care services with chronic diseases: a qualitative study of patient and family experience. Ann Fam Med 2015;13:523-8.

5. Bogner HR, Joo JH, Hwang S, et al. Does a depression management program decrease mortality in older adults with specific medical conditions in primary care? An exploratory analysis. J Am Geriatr Soc 2016;64:126-31.

6. Gallo JJ, Hwang S, Joo JH, et al. Multimorbidity, depression, and mortality in primary care: random- 
ized clinical trial of an evidence-based depression care management program on mortality risk. J Gen Intern Med 2016;31: 380-6.

7. Peikes DN, Reid RJ, Day TJ, et al. Staffing patterns of primary care practices in the comprehensive primary care initiative. Ann Fam Med 2014;12:142-9.

8. Howard H, Malouin R, Callow-Rucker M. Care managers and knowledge shift in primary care patient-centered medical home transformation. Hum Organ 2016;75:10-20.

9. Hines P, Mercury M. Designing the role of the embedded care manager. Prof Case Manag 2013;18: 182-7; quiz 188-9.

10. Smith, AC. Role ambiguity and role conflict in nurse case managers: an integrative review. Prof Case Manag 2011;16:182-96; quiz 197-8.

11. Luo Z, Chen Q, Annis AM, et al. A comparison of health plan- and provider-delivered chronic care management models on patient clinical outcomes. J Gen Intern Med 2016;31:762-70.

12. Annis AM, Holtrop JS, Tao M, Chang HC, Luo Z. Comparison of provider and plan-based targeting strategies for disease management. Am J Manag Care 2015;21:344-51.

13. Carayon P, Hundt AS, Hoonakker P, et al. Perceived impact of care managers' work on patient and clinician outcomes. Eur J Pers Cent Healthc 2015;3:15867.

14. Stille C, Turchi RM, Antonelli R, et al. The familycentered medical home: specific considerations for child health research and policy. Acad Pediatr 2010; 10:211-7.

15. Kellerman SE, Herold J. Physician response to surveys. A review of the literature. Am J Prev Med 2001;20:61-7.

16. Willis GB, Smith T, Lee HJ. Do additional recontacts to increase response rate improve physician survey data quality? Med Care 2013;51:945-8.

17. Holtrop JS, Potworowski G, Fitzpatrick L, Kowalk A, Green LA, et al. Effect of care management program structure on implementation: a normalization process theory analysis. BMC Health Serv Res 2016; $16(\mathrm{a}): 386$. 